\title{
M-Polynomials And Topological Indices Of Zigzag And Rhombic Benzenoid Systems
}

https://doi.org/10.1515/chem-2018-0010

received August 22, 2017; accepted December 4, 2017.

Abstract: M-polynomial of different molecular structures helps to calculate many topological indices. This polynomial is a new idea and its beauty is the wealth of information it contains about the closed forms of degreebased topological indices of molecular graph $G$ of the structure. It is a well-known fact that topological indices play significant role in determining properties of the chemical compound [1-4]. In this article, we computed the closed form of M-polynomial of zigzag and rhombic benzenoid systemsbecause of their extensive usages in industry. Moreover we give graphs of M-polynomials and their relations with the parameters of structures.

Keywords: M-polynomial, Topological index, Benzenoid.

\section{Introduction}

In mathematical chemistry, mathematical tools such as polynomials and numbers predict properties of compounds without using quantum mechanics. These tools, in combination, capture information hidden in the symmetry of molecular graphs. Most commonly known invariants of such kinds are known as degree-based topological indices. These are the numerical values that correlate the structure with various physical properties, chemical reactivity and biological activities [5-12]. It is an established fact that many properties such as heat of formation, boiling point, strain energy, rigidity and fracture toughness of a molecule are strongly connected

\footnotetext{
*Corresponding author: Shin Min Kang: Department of Mathematics and RINS, Gyeongsang National University, Jinju 52828, Korea; Center for General Education, China Medical University, Taichung 40402, Taiwan, E-mail: smkang@gnu.ac.kr

Ashaq Ali: Department of mathematics and statistics, The university of Lahore, Lahore Pakistan

Waqas Nazeer, Mobeen Munir: Division of Science and Technology, University of Education, Lahore, 54000, Pakistan
}

to its graphical structure and this fact plays a synergic role in chemical graph theory. A graph $G$ with vertex set $V(G)$ and edge set $E(G)$ is connected, if there exists a connection between any pair of vertices in $\mathrm{G}$. The distance between two vertices $u$ and $v$ is denoted as $d(u, v)$ and is the length of the shortest path between $u$ and $v$ in graph $G$. The number of vertices of $G$, adjacent to a given vertex $v$, is the "degree" of this vertex, and will be denoted by $d_{v}$. For details on basics of graph theory, any standard text such as [13] can be of great help.

Several algebraic polynomials haveuseful applications in chemistry such as Hosoya Polynomial (also called Wiener polynomial) [8]. M-polynomial [14], introduced in 2015 helps in determining many degree-based topological indices. Benzenoid hydrocarbons play a vital role in our environment, and in the food and chemical industries. Benzenoid molecular graphs are systems with deleted hydrogens. It is a connected geometric figure obtained by arranging congruent regular hexagons in a plane, so that two hexagons are either disjoint or have a common edge. This figure divides the plane into one infinite (external) region and a number of finite (internal) regions. All internal regions must be regular hexagons. Benzenoid systems are of considerable importance in theoretical chemistry because they are the natural graph representation of benzenoid hydrocarbons. A vertex of a hexagonal system belongs to, at most, three hexagons. A vertex shared by three hexagons is called an internal vertex. Under this definition in [15] the figure under discussion is not a benzenoid system as one internal central region is a regular 14 sided polygon. These systems are planar consisting of regular hexagons as shown in Figure 1.

Definition 1. Let $\mathrm{G}$ be a simple connected graph. The M-polynomial of $\mathrm{G}$ is defined as:

$$
M(G, x, y)=\sum_{\delta \leq i \leq j \leq \Delta} m_{i j}(G) x^{i} y^{j} .
$$

Where $\delta=\operatorname{Min}\left\{d_{v} \mid v \in \mathrm{V}(\mathrm{G})\right\}, \quad \Delta=\operatorname{Max}\left\{d_{v} \mid v \in \mathrm{V}(\mathrm{G})\right\}$, and $m_{i j}(G)$ is the edge $v u \in E(G)$ such that $\left\{d_{v}, d_{u}\right\}=\{i, j\}$.

This polynomial has been one of the key areas of interest in computational aspects of materials. From 

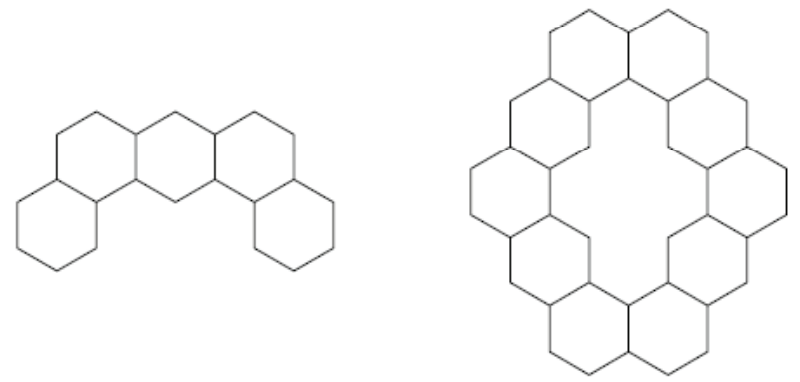

Figure 1: Example of benzenoid system left and a non - benzenoid system right.

this M-polynomial, we can calculate many topological indices. The topological index of a molecule structure can be considered as a non-empirical numerical quantity which quantifies the molecular structure and its branching pattern in many ways. M-polynomial of different molecular structures have been computed in $[9,10,12,16,17$,$] . Essentially , the topological index can be$ regarded as a score function which maps each molecular structure to a real number and is used as a descriptor of the molecule under testing [1,18-20]. Topological indices provide a good prediction of various physico-chemical properties of chemical compounds including boiling point, heat of evaporation, heat of formation, chromatographic retention times, surface tension, vapor pressure etc. Since the 1970s, two degree based graph invariants have been extensively studied. These are the first Zagreb index $M_{1}$ and the second Zagreb index $M_{2}$, introduced by Gutman and Trinajstic' [2] and defined as:

$$
M_{1}(G)=\sum_{v \in V(G)}\left(\mathrm{d}_{v}\right)^{2} \text { and } M_{2}(G)=\sum_{u v \in E(G)} \mathrm{d}_{u} \mathrm{~d}_{v} .
$$

Results obtained in the theory of Zagreb indices are summarized in the review [21].

Second modified Zagreb index is defined as:

$$
{ }^{m} M_{2}(G)=\sum_{u v \in E(G)} \frac{1}{d_{u} d_{v}} .
$$

In 1998, working independently, Bollobas and Erdos [22] and Amic et al. [3] proposed general Randic index. It has been extensively studied by both mathematicians and theoretical chemists

(See, for example, [23]). The Randic' index is defined as:

$$
R_{\alpha}(G)=\sum_{u v \in E(G)}\left(d_{u} d_{v}\right)^{\alpha},
$$

where $\alpha$ is an arbitrary real number.
Table 1: Derivation of some degree-based topological indices from M-polynomial.

\begin{tabular}{ll}
\hline $\begin{array}{l}\text { Topological Index } \\
\text { Second Modified Zagreb }\end{array}$ & $\begin{array}{l}\text { Derivation from } \mathbf{M}(\mathbf{G} ; \mathbf{x}, \mathbf{y}) \\
\left.\left(S_{x} S_{y}\right)(M(G ; x, y))\right|_{x=y=1} \\
\text { General Randić }\end{array}$ \\
$\begin{array}{l}\left.\left(D_{x}^{\alpha} D_{y}^{\alpha}\right)(M(G ; x, y))\right|_{x=y=1} \\
\text { General Randić }\end{array}$ & $\left.\left(S_{x}^{\alpha} S_{y}^{\alpha}\right)(M(G ; x, y))\right|_{x=y=1}$ \\
Symmetric Division Index & $\left.\left(D_{x} S_{y}+S_{x} D_{y}\right)(M(G ; x, y))\right|_{x=y=1}$ \\
Harmonic Index & $2 S_{x} J(M(G ; x, y))_{x=1}$ \\
Inverse sum Index & $S_{x} J D_{x} D_{y}(M(G ; x, y))_{x=1}$ \\
Augmented Zagreb Index & $S_{x}{ }^{3} Q_{-2} J D_{x}{ }^{3} D_{y}{ }^{3}(M(G ; x, y))_{x=1}$ \\
\hline Where & $\partial(f(x, y)$ \\
$D_{x}=x \frac{\partial(f(x, y)}{\partial x}, D_{y}=y \frac{x}{\partial y}, \frac{f(t, y)}{t} d t, S_{y}=$ \\
$y$ \\
$\int_{0}^{y} \frac{f(x, t)}{t} d t, J(f(x, y))=f(x, x), Q_{\alpha}(f(x, y))=x^{\alpha} f(x, y)$.
\end{tabular}

Symmetric division index is defined as:

$S D D(G)=\sum_{u v \in E(G)}\left\{\frac{\min \left(d_{u}, d_{v}\right)}{\max \left(d_{u}, d_{v}\right)}+\frac{\max \left(d_{u}, d_{v}\right)}{\min \left(d_{u}, d_{v}\right)}\right\}$.

Another variant of Randic' index is the harmonic index defined as:

$H(G)=\sum_{v u \in E(G)} \frac{2}{d_{u}+d_{v}}$.

The Inverse sum index is defined as:

$$
I(G)=\sum_{v u \in E(G)} \frac{d_{u} d_{v}}{d_{u}+d_{v}} .
$$

The augmented Zagreb index is defined as:

$$
A(G)=\sum_{v u \in E(G)}\left\{\frac{d_{u} d_{v}}{d_{u}+d_{v}-2}\right\}^{3},
$$

and it is useful for computing heat of formation of alkanes $[24,25]$.

For detailed study about degree-based topological indices, we refer [26-32] and the references therein.

These topological indices can be recovered from M-polynomial [14], see following Table 1.

In this article, we compute the closed form of the M-polynomial for two famous benzenoid systems Zigzag benzenoid system and Rhombic benzenoid system. We also computed some degree-based topological indices. 


\section{Methodology}

At first we obtain general pattern of vertex and edge partitions of 2D molecular graph connected to the Zigzag benzenoid system and Rhombic benzenoid system based on the degree of end vertices of edges. From this edge partition, using definition, we obtain M-polynomials of these systems. The 3D graph of M-polynomials are sketched by using maple 2015. Then using mathematical operators and table 1 , we reach at the different degreebased indices.

Ethical approval: The conducted research is not related to either human or animals use.

\section{Results and Discussions}

In this part we give our main computational results in two sections.

\subsection{Computational aspects of Zigzag benzenoid system}

Let $n$ be number of rows in graph of zigzag benzenoid system $Z_{n}$ with two hexagons in each row. Since first row contain two hexagons with twelve edges and one edge is common so we obtain total eleven edges in the first row and combining first and second row we obtain 24 total edges with three edges in common so we obtain 21 different edges. Continuing in the same way we obtain $10 n+1$ edges and $8 n+2$ vertices. We partition edges on the basis of degrees of endpoints of edges of the graph. All vertices are either of degree two or three. One can observe that at each row we have two edges of type $\{2,2\}$, one upside and one downside the chain except the endpoints where we have two more edges with end vertex having degree two.

Thus $\left|E_{\{2,2\}}\right|=\left|\left\{e=u v \in E\left(Z_{n}\right) \mid d_{u}=2, d_{v}=2\right\}\right|=2 n+4$. Next we can see that four edges of type $(2,3)$ are present in each row of the chain so $\left|E_{\{2,3\}}\right|=\mid\left\{e=u v \in E\left(Z_{n}\right) \mid d_{u}=\right.$ $\left.=2, d_{v}=3\right\}=4 n$. Remaining are edges of type $(3,3)$ given as . $\left|E_{\{, 3,3\}}\right|=\left|\left\{e=u v \in E\left(Z_{n}\right) \mid d_{u}=3, d_{v}=3\right\}\right|=(10 n+1)-$ $-(2 n+4)-4 n=4 n-3$.

Theorem 1. Consider the zigzag benzenoid system $Z_{n}$, then its M-polynomial is

$M\left(Z_{n} ; x, y\right)=2(n+2) x^{2} y^{2}+4 n x^{2} y^{3}+(4 n-3) x^{3} y^{3}$.

Proof. Let $Z_{n}$ be the zigzag benzenoid system, then from the above decision $\left|V\left(Z_{n}\right)\right|=8 \mathrm{n}+2$ and $\left|E\left(Z_{n}\right)\right|=10 \mathrm{n}+1$. Also from the above decision, we can divide the edge set

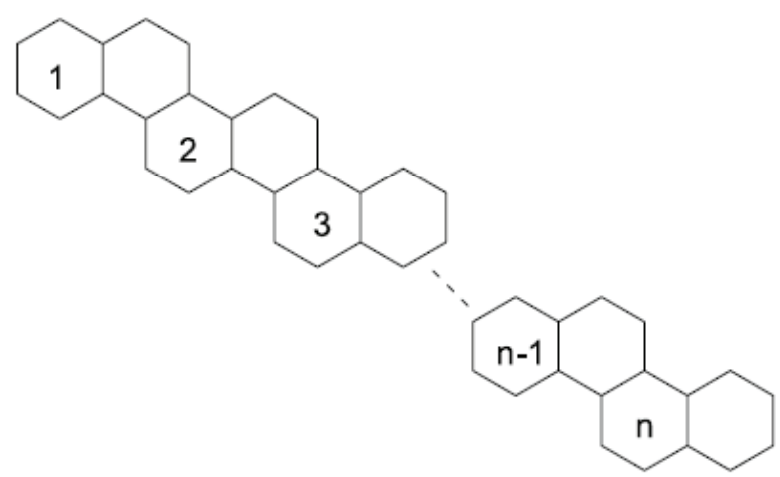

Figure 2: Graph of zigzag benzenoid system $Z_{n}$.

into the following three partitions:

$E_{1}\left(Z_{n}\right)=\left\{e=u v \in E\left(Z_{n}\right): \quad d_{u}=d_{v}=2\right\}$,

$E_{2}\left(Z_{n}\right)=\left\{e=u v \in E\left(Z_{n}\right): \quad d_{u}=2, d_{v}=3\right\}$

$E_{3}\left(Z_{n}\right)=\left\{e=u v \in E\left(Z_{n}\right): \quad d_{u}=d_{v}=3\right\}$.

In addition,

$\left|E_{1}\left(Z_{n}\right)\right|=2(\mathrm{n}+2)$,

$\left|E_{2}\left(Z_{n}\right)\right|=4 n$,

$\left|E_{3}\left(Z_{n}\right)\right|=4 n-3$.

Now by definition of M-polynomial, we have

$$
\begin{aligned}
M\left(Z_{n} ; x, y\right) & =\sum_{i \leq j} m_{i j} x^{i} y^{j} \\
& =\sum_{2 \leq 2} m_{22} x^{2} y^{2}+\sum_{2 \leq 3} m_{23} x^{2} y^{3}+\sum_{3 \leq 3} m_{33} x^{3} y^{3} \\
& =\sum_{u v \in E_{1}\left(Z_{n}\right)} m_{22} x^{2} y^{2}+\sum_{u v \in E_{2}\left(Z_{n}\right)} m_{23} x^{2} y^{3}+\sum_{u v \in E_{3}\left(Z_{n}\right)} m_{33} x^{3} y^{3} \\
& =\left|E_{1}\left(Z_{n}\right)\right| x^{2} y^{2}+\left|E_{2}\left(Z_{n}\right)\right| x^{2} y^{3}+\left|E_{3}\left(Z_{n}\right)\right| x^{3} y^{3} \\
& =2(n+2) x^{2} y^{2}+4 n x^{2} y^{3}+(4 n-3) x^{3} y^{3} .
\end{aligned}
$$

Now we compute some degree-based topological indices of zigzag benzenoid from this M-polynomial.

Proposition 2. Consider the zigzag benzenoid system $Z_{n}$, then

1. ${ }^{m} M_{2}\left(Z_{n}\right)=\frac{29}{18} n+\frac{2}{3}$

2. $R_{\alpha}\left(Z_{n}\right)=4 n 9^{\alpha}+2 n 4^{\alpha}+4 n 6^{\alpha}-3 \times 9^{\alpha}+4 \times 4^{\alpha}$.

3. $R_{\alpha}\left(Z_{n}\right)=\frac{2 n+4}{4^{\alpha}}+\frac{4 n}{6^{\alpha}}+\frac{4 n-3}{9^{\alpha}}$.

4. $\operatorname{SSD}\left(Z_{n}\right)=\frac{62}{3} n+2$.

5. $H\left(Z_{n}\right)=\frac{31}{3} n+1$.

6. $I\left(Z_{n}\right)=\frac{64}{5} n-\frac{1}{2}$ 


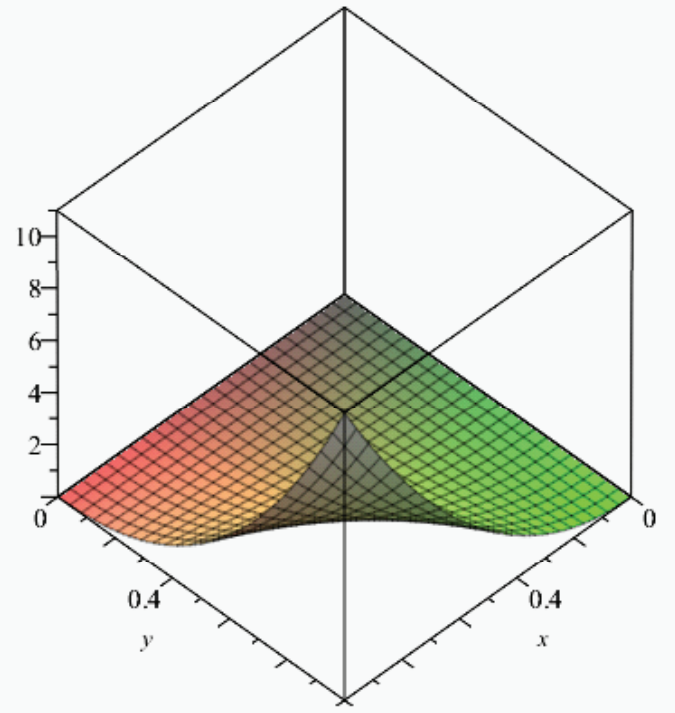

Figure 3: The plot for the M-polynomial of $Z_{1}$.

7. $A\left(Z_{n}\right)=\frac{1457}{16} n-\frac{459}{64}$.

Proof. Let $M\left(Z_{n} ; x, y\right)=f(x, y)=2(n+2) x^{2} y^{2}+4 n x^{2} y^{3}+(4 n-3) x^{3} y^{3}$. Then

$$
\begin{aligned}
& D_{x} f(x, y)=4(n+2) x^{2} y^{2}+8 n x^{2} y^{3}+3(4 n-3) x^{3} y^{3}, \\
& D_{y} f(x, y)=4(n+2) x^{2} y^{2}+12 n x^{2} y^{3}+3(4 n-3) x^{3} y^{3}, \\
& D_{y} D_{x} f(x, y)=8(n+2) x^{2} y^{2}+24 n x^{2} y^{3}+9(4 n-3) x^{3} y^{3}, \\
& S_{y}(f(x, y))=(n+2) x^{2} y^{2}+\frac{4}{3} n x^{2} y^{3}+\frac{1}{3}(4 n-3) x^{3} y^{3}, \\
& S_{x} S_{y}(f(x, y))=\frac{1}{2}(n+2) x^{2} y^{2}+\frac{2}{3} n x^{2} y^{3}+\frac{1}{9}(4 n-3) x^{3} y^{3}, \\
& D_{y}{ }^{\alpha}(f(x, y))=2^{\alpha+1}(n+2) x^{2} y^{2}+3^{\alpha} 4 n x^{2} y^{3}+3^{\alpha}(4 n-3) x^{3} y^{3}, \\
& D_{x}^{\alpha} D_{y}^{\alpha}(f(x, y))=2^{2 \alpha+1}(n+2) x^{2} y^{2}+2^{\alpha+2} 3^{\alpha} n x^{2} y^{3}+3^{2 \alpha}(4 n-3) x^{3} y^{3}, \\
& S_{y}^{\alpha}(f(x, y))=\frac{1}{2^{\alpha-1}}(n+2) x^{2} y^{2}+\frac{4}{3^{\alpha}} n x^{2} y^{3}+\frac{1}{3^{\alpha}}(4 n-3) x^{3} y^{3}, \\
& S_{x}^{\alpha} S_{y}^{\alpha}(f(x, y))=\frac{1}{2^{2 \alpha-1}}(n+2) x^{2} y^{2}+\frac{1}{2^{\alpha-2} 3^{\alpha}} n x^{2} y^{3}+\frac{1}{3^{2 \alpha}}(4 n-3) x^{3} y^{3}, \\
& S_{y} D_{x}(f(x, y))=2(n+2) x^{2} y^{2}+\frac{8}{3} n x^{2} y^{3}+(4 n-3) x^{3} y^{3}, \\
& S_{x} D_{y}(f(x, y))=2(n+2) x^{2} y^{2}+6 n x^{2} y^{3}+(4 n-3) x^{3} y^{3}, . \\
& J f(x, y)=2(n+2) x^{4}+4 n x^{5}+(4 n-3) x^{6}, \\
& S_{x} J f(x, y)=\frac{1}{2}(n+2) x^{4}+\frac{4}{5} n x^{5}+\frac{1}{6}(4 n-3) x^{6},
\end{aligned}
$$

$$
\begin{aligned}
& J D_{x} D_{y} f(x, y)=8(n+2) x^{4}+24 n x^{5}+9(4 n-3) x^{6}, \\
& S_{x} J D_{x} D_{y} f(x, y)=2(n+2) x^{4}+\frac{24}{5} n x^{5}+\frac{3}{2}(4 n-3) x^{6}, \\
& D_{y}{ }^{3} f(x, y)=16(n+2) x^{2} y^{2}+108 n x^{2} y^{3}+27(4 n-3) x^{3} y^{3}, \\
& D_{x}{ }^{3} D_{y}{ }^{3} f(x, y)=108(n+2) x^{2} y^{2}+864 n x^{2} y^{3}+729(4 n-3) x^{3} y^{3}, \\
& J D_{x}{ }^{3} D_{y}{ }^{3} f(x, y)=108(n+2) x^{4}+864 n x^{5}+729(4 n-3) x^{6}, \\
& Q_{-2} J D_{x}{ }^{3} D_{y}{ }^{3} f(x, y)=108(n+2) x^{2}+864 n x^{3}+729(4 n-3) x^{4}, \\
& S_{x}{ }^{3} Q_{-2} J D_{x}{ }^{3} D_{y}{ }^{3} f(x, y)=\frac{27}{2}(n+2) x^{2}+32 n x^{3}+\frac{729}{64}(4 n-3) x^{4} .
\end{aligned}
$$$$
\text { 1. }{ }^{m} M_{2}\left(Z_{n}\right)=\left.S_{x} S_{y}(f(x, y))\right|_{x=y=1}=\frac{29}{18} n+\frac{2}{3} .
$$$$
\text { 2. } R_{\alpha}\left(Z_{n}\right)=\left.D_{x}^{\alpha} D_{y}^{\alpha}(f(x, y))\right|_{x=y=1}=4 n 9^{\alpha}+2 n 4^{\alpha}+4 n 6^{\alpha}-3 \times 9^{\alpha}+4 \times 4^{\alpha} \text {. }
$$$$
\text { 3. } R_{\alpha}\left(Z_{n}\right)=\left.S_{x}^{\alpha} S_{y}^{\alpha}(f(x, y))\right|_{x=y=1}=\frac{2 n+4}{4^{\alpha}}+\frac{4 n}{6^{\alpha}}+\frac{4 n-3}{9^{\alpha}} \text {. }
$$$$
\text { 4. } \operatorname{SSD}\left(Z_{n}\right)=\left.\left(S_{y} D_{x}+S_{x} D_{y}\right)(f(x, y))\right|_{x=y=1}=\frac{62}{3} n+2 \text {. }
$$$$
\text { 5. } H\left(Z_{n}\right)=\left.2 S_{x} J(f(x, y))\right|_{x=1}=\frac{31}{3} n+1 .
$$$$
\text { 6. } I\left(Z_{n}\right)=S_{x} J D_{x} D_{y}(f(x, y))_{x=1}=\frac{64}{5} n-\frac{1}{2} .
$$$$
\text { 7. } A\left(Z_{n}\right)=\left.S_{x}{ }^{3} Q_{-2} J D_{x}{ }^{3} D_{y}{ }^{3}(f(x, y))\right|_{x=1}=\frac{1457}{16} n-\frac{459}{64} \text {. }
$$

\subsection{Computational aspects of the Rhombic benzenoid system}

Take another benzenoid system in which hexagons are arranged to form a rhombic shape $R_{n}$, in which there are $n$ rows of $n$ hexagons as given in Figure 4. Then it has $2 n(n+2)$ vertices and $3 n^{2}+4 n-1$ edges. On the similar lines we partition edges on the basis of degrees of endpoints. Clearly, all vertices are either of $2^{\text {nd }}$ or 3 rd degree. $E_{\{2,2\}}$ appear only on the four corners of the rhomb, so $\left|E_{\{2,2\}}\right|=\left|\left\{e=u v \in E\left(R_{n}\right) \mid d_{u}=2, d_{v}=2\right\}\right|=6$. Edges $E_{\{2,3\}}$ appear in pairs along the boundary except the corners where they are single. So we obtain $\left|E_{\{2,3\}}\right|=\left|\left\{e=u v \in E\left(Z_{n}\right) \mid d_{u}=2, d_{v}=3\right\}\right|=8(n-1)$. Remaining are edges of type $(3,3)$ given as $\left|E_{\{\{, 3\}}\right|=\left|\left\{e=u v \in E\left(Z_{n}\right) \mid d_{u}=3, d_{v}=3\right\}\right|=3 n^{2}+4 n-1-1$ $-6-8(n-1)=3 n^{2}-4 n+1$.

So we obtain the following result.

Theorem 3. Consider the rhombic benzenoid system $R_{n}$, then its M- polynomial is:

$M\left(R_{n} ; x, y\right)=6 x^{2} y^{2}+8(n-1) x^{2} y^{3}+(n(3 n-4)+1) x^{3} y^{3}$. 


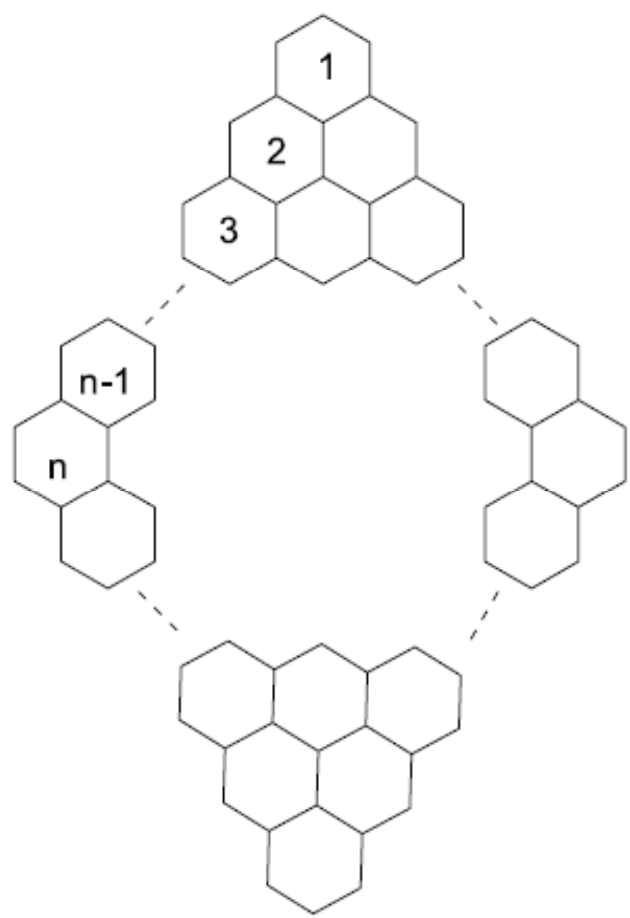

Figure 4: Graph of rhombic benzenoid system having $n$ rows of $n$ hexagons.

Proof. The proof is similar to theorem 1.

Now we compute some degree-based topologcal indices from this M-polynomial.

Proposition 4. Consider the rhombic benzenoid system $R_{n}$, then

1. ${ }^{m} M_{2}\left(R_{n}\right)=\frac{5}{18}+\frac{4}{3} n+\frac{1}{9} n(3 n-4)$.

2. $R_{\alpha}\left(R_{n}\right)=9^{\alpha} n(3 n-4)+8 n 6^{\alpha}+9^{\alpha}+6 \times 4^{\alpha}-8 \times 6^{\alpha}$.

3. $R_{\alpha}\left(R_{n}\right)=\frac{6}{4^{\alpha}}+\frac{8 n-8}{6^{\alpha}}+\frac{n(3 n-4)+1}{9^{\alpha}}$.

4. $\operatorname{SSD}\left(R_{n}\right)=-\frac{10}{3}+\frac{52}{3} n+2 n(3 n-4)$.

5. $H\left(R_{n}\right)=\frac{2}{15}+\frac{16}{5} n+\frac{1}{3} n(3 n-4)$.

6. $I\left(R_{n}\right)=-\frac{21}{10}+\frac{48}{5} n+\frac{3}{2} n(3 n-4)$.

7. $A\left(R_{n}\right)=-\frac{295}{64}+64 n+\frac{729}{64} n(3 n-4)$.

Proof. The proof of this proposition is similar to proposition 2.

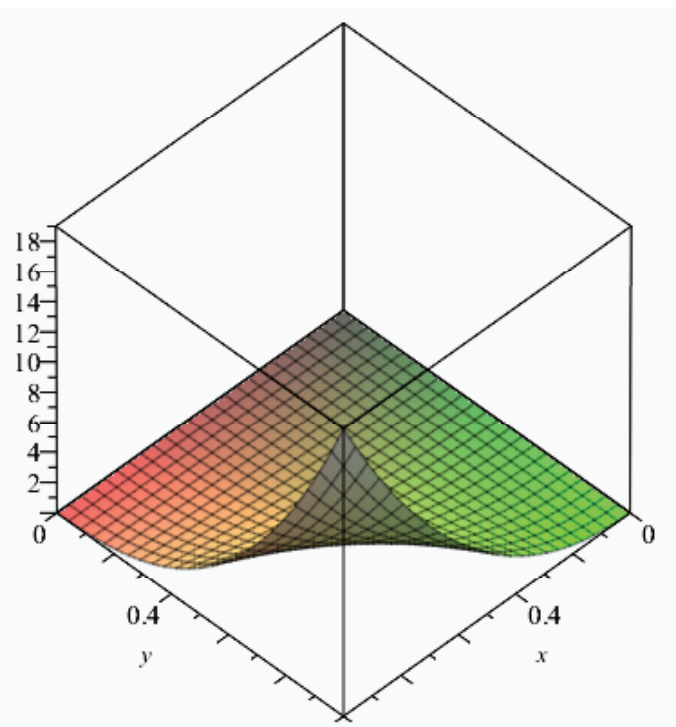

Figure 6: The plot for the M-polynomial of $R_{1}$.

\section{Conclusions and Discussion}

In this article we computed M-polynomials and closed forms of degree-based topological indices of zigzag and rhombic benzenoid system. These indices play important role in determining properties of compound under investigations. We gave graphs of M-polynomials against the number of hexagons $n$ in each structure. These graphs in fact determine the dependency of above discussed topological indices relating to $\mathrm{n}$.

Acknowledgments: Authors are highly grateful to the referees for their careful reading and technical suggestions to improve the quality of the article. This research is supported by Gyeongsang National University, Jinju 52828, Korea

Author Contributions: All authors contribute equally in writing of this paper.

Conflicts of Interest: The authors declare no conflict of interest.

\section{References}

[1] Rücker G., Rücker C., On topological indices, boiling points, and cycloalkanes. Journal of chemical information and computer sciences. 1999, 39(5),788-802. 
[2] Gutman I,, Trinajstić N., Graph theory and molecular orbitals. Total $\varphi$-electron energy of alternant hydrocarbons. Chemical Physics Letters. 197, 17(4), 535-8.

[3] Amić D., Bešlo D., Lučić B., Nikolić S., Trinajstić N., The vertexconnectivity index revisited. Journal of chemical information and computer sciences. 1998, 38(5), 819-822.

[4] Kier L.B., Hall L.H., Molecular connectivity in structure-activity analysis. Research Studies, 1986.

[5] Gutman I., Molecular graphs with minimal and maximal Randić indices. Croatica chemica acta, 2002, 75(2), 357-369.

[6] Gutman I., Degree-based topological indices. Croatica Chemica Acta, 2013, 86(4), 351-361.

[7] Vuki cevi 'c, D., On the edge degrees of trees. Glas. Mat. Ser. III, 2009, 44(64), 259-266.

[8] Dobrynin A.A., Entringer R., Gutman I., Wiener index of trees: theory and applications. Acta Applicandae Mathematicae, 2001, 66(3), 211-249.

[9] Munir M., Nazeer W., Rafique S., Kang S.M., M-polynomial and degree-based topological indices of polyhex nanotubes. Symmetry, 2016, 8(12), 149.

[10] Munir M., Nazeer W., Rafique S., Kang S.M., M-polynomial and degree-based topological indices of polyhex nanotubes. Symmetry, 2016, 8(12),149.

[11] Ajmal M., Kang S.M., Nazeer W., Munir M., Jung C.Y., Some Topological Invariants of the Möbius Ladders. Global Journal of Pure and Applied Mathematics, 2016, 12(6), 5317-5327.

[12] Munir M., Nazeer W., Rafique S., Nizami A., Kang S. M., Some Computational Aspects of Triangular Boron Nanotubes, Symmetry, 2017, 9, 6, doi:10.3390/sym9010006.

[13] West D.B., Introduction to Graph Theory Prentice Hall Upper Saddle River. NJ Google Scholar, 1996.

[14] Deutsch E., Klavzar S., M-Polynomial and Degree-Based Topological Indices. Iranian Journal of Mathematical Chemistry, 2015, 6(2), 93-102.

[15] Wu R., Deng H., The general connectivity indices of benzenoid systems and phenylenes. MATCH Commun. Math. Comput. Chem., 2010, 64, 459-470.

[16] Javaid M., Jung C. Y., M-Polynomials and Topological Indices of Silicate and Oxide Networks, International Journal of Pure and Applied Mathematics, 2017, 115(1), 129-152, doi: 10.12732/ ijpam.v115i1.11

[17] Kwun Y.C., Munir M., Nazeer W., Rafique S., Kang S.M., M-Polynomials and topological indices of V-Phenylenic Nanotubes and Nanotori. Scientific reports, 201, 7(1), 8756.

[18] Klavžar S., Gutman I., A comparison of the Schultz molecular topological index with the Wiener index. Journal of chemical information and computer sciences. 1996 Sep 24;36(5):10011003.

[19] Deng H., Huang G., Jiang X., A unified linear-programming modeling of some topological indices. Journal of Combinatorial Optimization, 2015, 30(3), 826-837.

[20] Deng H., Yang J., Xia F., A general modeling of some vertexdegree based topological indices in benzenoid systems and phenylenes. Computers \& Mathematics with Applications, 2011, 61(10), 3017-23.

[21] Gutman I., Das K.C., The first Zagreb index 30 years after. MATCH Commun. Math. Comput. Chem., 2004, 50, 83-92.

[22] Bollobás B., Erdös P., Graphs of extremal weights. Ars Combinatoria, 1998, 50, 225-233.
[23] Kier L.B., Hall L.H., Molecular connectivity in structure-activity analysis. Research Studies, 1986.

[24] Huang Y., Liu B., Gan L., Augmented Zagreb index of connected graphs. Match-Communications in Mathematical and Computer Chemistry, 2012, 67(2), 483-494.

[25] Furtula B., Graovac A., Vukicevic D., Augmented Zagreb index, J. Math. Chem., 2010, 48, 370-380.

[26] Farahani M.R., Rajesh Kanna M.R., Jamil M.K., Imran M., Computing the M-Polynomial of Benzenoid Molecular Graphs. Science International (Lahore), 2016, 28, 3251-3255.

[27] Sardar M. S., Zafar S., Farahani M.R., THE GENERALIZED ZAGREB INDEX OF CAPRA-DESIGNED PLANAR BENZENOID SERIES \$Ca_k(C_6)\$, Open J. Math. Sci., 2017, $144-51$.

[28] Mutee ur Rehman H., Sardar R., Raza A., Computing Topological Indices of Hex Board and its Line Graph, Open J. Math. Sci., 2017, 1, 62 - 71.

[29] Sardar, M. S., Pan X.X., Gao W., Farahani M. R., Computing Sanskruti Index of Titania Nanotubes, Open J. Math. Sci., 2017, $126-131$

[30] Farahani M.R., Gao W., Baig A.Q., Khalid W., Molecular description of copper (II) oxide. Macedonian Journal of Chemistry and Chemical Engineering, 2017, 36(1), 93-99.

[31] Gao W., Wang Y., Wang W., Shi L., The first multiplication atombond connectivity index of molecular structures in drugs. Saudi Pharmaceutical Journal, 2017, 25(4), 548-555.

[32] Gao W., Farahani M.R., Wang S., Husin M.N., On the edgeversion atom-bond connectivity and geometric arithmetic indices of certain graph operations. Applied Mathematics and Computation, 2017, 308, 11-17. 